\title{
Curvature Matched Machining Methods Versus Commercial CAD Methods
}

\author{
K. A. White \\ Contour Numerical Control, Inc., Provo, Utah \\ J. K. Hill and C. G. Jensen \\ Mechanical Engineering, Brigham Young University, Provo, Utah
}

\begin{abstract}
This paper examines the benefits that are realized when five-axis curvature matched machining methods (CM) are used to machine typical automobile stamping die geometry. Three-axis ball end mill methods are used as the baseline of comparison for CM. Two doubly curved sample surfaces which represent an automotive stamping die are machined using: 1) three-axis ball end mill CAD methods, 2) $\mathrm{CM}$ methods developed by Brigham Young University and Contour Numerical Control, Inc. Results are presented and discussed.
\end{abstract}

\section{INTRODUCTION}

Traditionally, sculptured surfaces have been machined with ball end cutters on three-axis mills. All of the leading Computer-Aided Design and Manufacturing (CAD/CAM) systems have well developed three-axis ball end machining capabilities. Three-axis ball end machining has been thoroughly researched, and most of the pertinent machining issues are well understood. Ball end machining is still the predominant choice in industry today for the machining of complex surfaces.

However, five-axis mills have become relatively common, and offer new approaches for sculptured surface machining. The most common approach is to use a flat end cutter inclined at a fixed angle from the surface or surface normal. A flat end cutter removes material much more efficiently than a ball end cutter of the same size, but fixing the tool angle introduces a series of problems not generally encountered in three-axis machining. The most severe problem occurs when the cutter is not inclined far enough from the surface and cutting occurs on the lateral and trailing edges of the cutter. This problem is known as local gouging. This leads to too much material removal as well as surface finish problems. Largely because of the gouging problem created by a fixed inclination, industry has not widely adopted five-axis machining methods for complex surfaces. To the author's knowledge, none of the currently available five-axis machining software algorithms found in CAD/CAM systems support gouge detection and correction for flat end cutters.

A new approach for machining with a flat or filleted end cutter is curvature matched machining $(\mathrm{CM})$. In this method the curvature of the surface at a discrete point is used to calculate the inclination angle of the cutter so that the curvature of the cutter matches the curvature of the surface. As the cutter moves across the surface, the inclination is automatically adjusted to maximize efficiency and eliminate gouging. This method results in a second order match between the surface and cutter, as opposed to the first order match created with three-axis machining or fixed-inclination five-axis machining, and leads to a significant increase in the material removal rate.

This paper compares the commercial implementation of $\mathrm{CM}$ developed by Contour Numerical Control, Inc. with machining algorithms found in three popular CAD/CAM systems: CATIA ${ }^{\circledR}$ by Dassault Systèmes, Pro/MANUFACTURE ${ }^{\text {TM }}$ by Parametric Technology Corporation and I-DEAS ${ }^{\text {TM }}$ by Structural Dynamics Research Corporation. Because of the limitations of fixed cutter inclination found in the commercial five-axis machining software tested here, CM was compared with three-axis ball end methods, which still represent the state-of-the-art in sculptured surface machining performed in industry today. Three-axis tool

The original version of this chapter was revised: The copyright line was incorrect. This has been corrected. The Erratum to this chapter is available at DOI: 10.1007/978-0-387-35392-0_40 
paths were generated in each of the previously mentioned $\mathrm{CAD} / \mathrm{CAM}$ systems and found to be essentially identical. Therefore, only the tool paths created in CATIA ${ }^{\circledR}$ were used for testing, since it is has the largest number of seats in the automotive/aerospace industries. Section 2 outlines the basic aspects of curvature matched machining. Section 3 describes the surfaces machined including all machining parameters. includes the basis for comparison and the judgment criteria and is followed by the results in section 4 . Conclusions are found in section 5 of this paper.

\section{MACHINING ALGORITHMS}

Ball end machining is very well understood and will not be described here. Curvature matched machining is a relatively new technology, and although it has been introduced in other publications (Mullins et al., 1993), it will be briefly discussed here.

The basic premise of CM is to match the curvature of the swept profile of the cutting tool to the normal curvature of the surface in the plane perpendicular to tool motion. Given a flat end mill, its swept silhouette is an ellipse. The major radius of the ellipse is equal to the tool radius, and the minor radius is a function of tool inclination. The minor radius varies from zero when the tool has no inclination to the tool radius, when the tool is inclined 90 degrees. The equation for the ellipse is

$$
\frac{x^{2}}{R^{2}}+\frac{y^{2}}{(R / \sin (\alpha))^{2}}=1
$$

(1)

It can be shown that the curvature of this ellipse at the cutter contact point is equal to

$$
\kappa=\frac{\sin (\alpha)}{R}
$$

(2)

Normal curvature on the surface, $n$, can be easily calculated from equations found in differential geometry theory. When set equal to the curvature of the cutter swept silhouette, an equation for the angle of inclination is found:

(3)

$$
\alpha=a \sin \left(\kappa_{n} * R\right)
$$

In $\mathrm{CM}$, the tool is inclined forward in the direction of tool motion by at each discrete tool location. A similar derivation for filleted end mills leads to an equation for inclination which will not be discussed here for sake of brevity.

\section{MACHINING METHODOLOGY}

For this comparison, two surfaces were machined, each one using CM and CATIA ${ }^{\circledR}$. The surfaces were the top and bottom of an automobile hood, representing a typical stamping die. This surface is displayed in Figure 1.

For this comparison, CATIA ${ }^{\circledR}$ used ball end cutters in three-axis mode while Contour Numerical Control's CM used a filleted end cutter in five-axis mode. Only finish passes were considered. For the top side of the hood one inch cutters (the filleted end cutter had a 0.125 in. corner radius) were used and for the underside of the hood half-inch cutters (the filleted end cutter had a 0.060 in. corner radius) were used. Scallop height was set to be a maximum of $0.0001 \mathrm{in}$. The surfaces were machined in aluminum. 


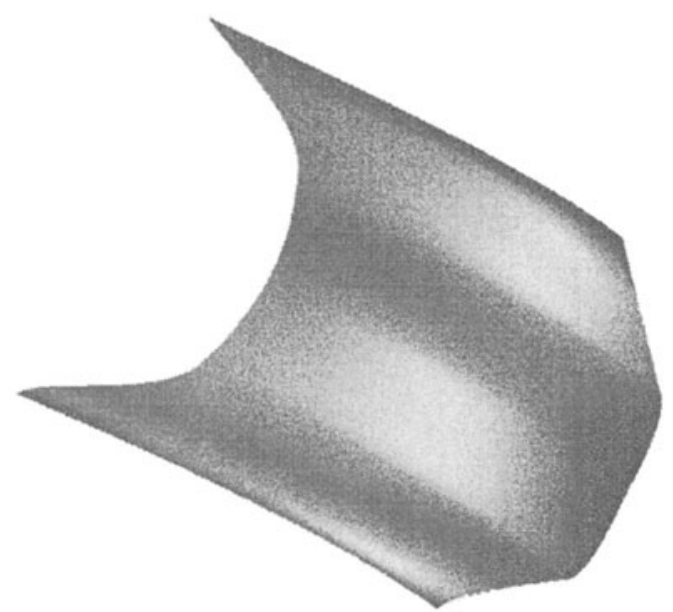

Figure 1: Hood surface.

\section{RESULTS}

The following figures are photographs of the machined surfaces set side-by-side. Upon close inspection, one can see the much wider step-over that occurred on the surfaces machined with $\mathrm{CM}$.

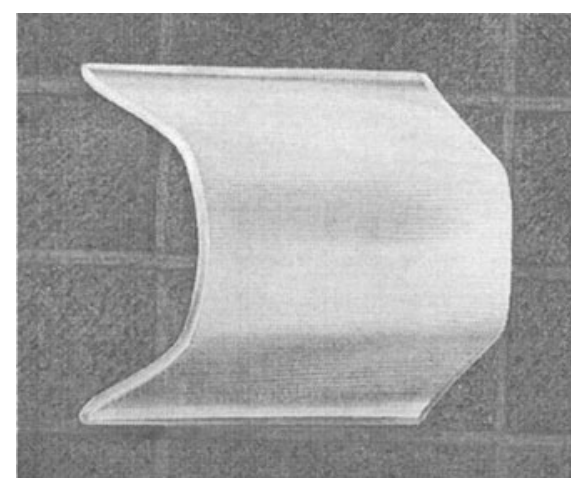

Figure 2: CM machined bottom of the hood.

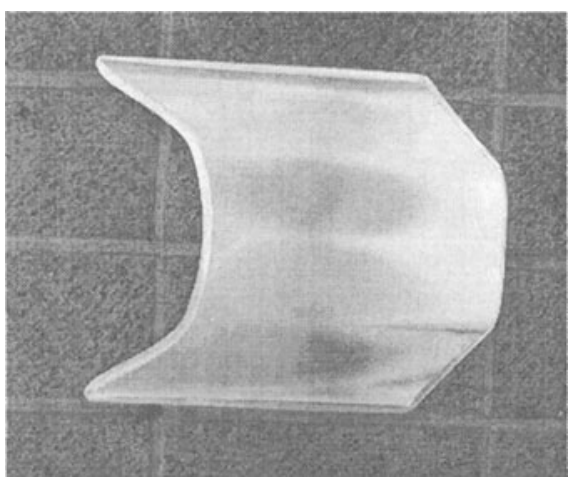

Figure 3: Ball end machined bottom of the hood.

The next figures are taken from a 3D scan performed by a Hommel T8000 surface analyzer. The same surface location was scanned on both surfaces. These scans were performed on a convex region on the top of the hood. The $y$-axis is scaled differently from the $\mathrm{x}$-axis in these scans so that surface features are more apparent. On the ball end portion, the individual scallops are clearly visible. On the CM portion, the step-over is much larger, and the scallops also appear to be smaller.

The next figure was also created using the Hommel surface analyzer. This figure was created by dragging the stylus across the scallops in a concave region on the top surface. The stroke length was 0.19 in, and the $y$-axis is scaled significantly larger than the $x$-axis so that scallops are easily discernible. In this graph, the scallops of the ball end mill are very easy to 
recognize, whereas scallops on the curvature matched portion are not easy to discern. The stroke on this graph covered approximately 2 adjacent tool paths but no distinct scallop is visible.

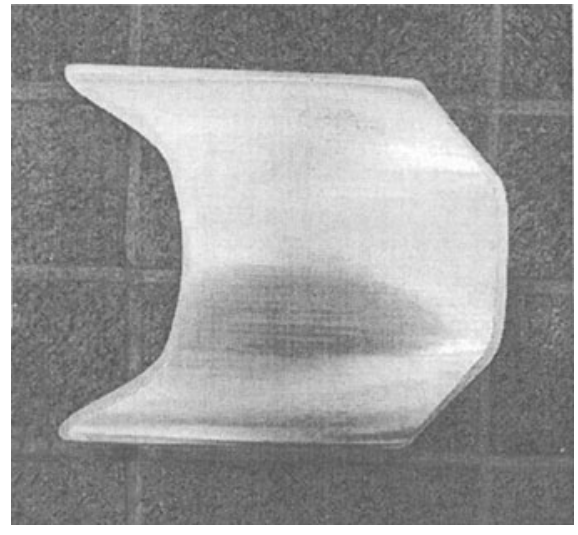

Figure 4: CM machined top of the hood.

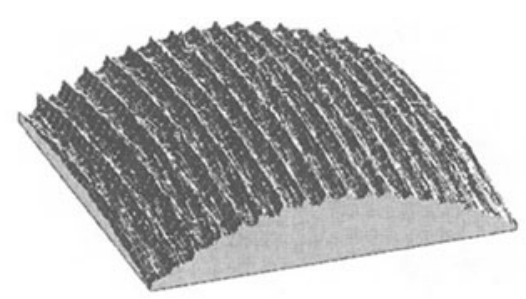

Figure 6: Ball end machined surface.

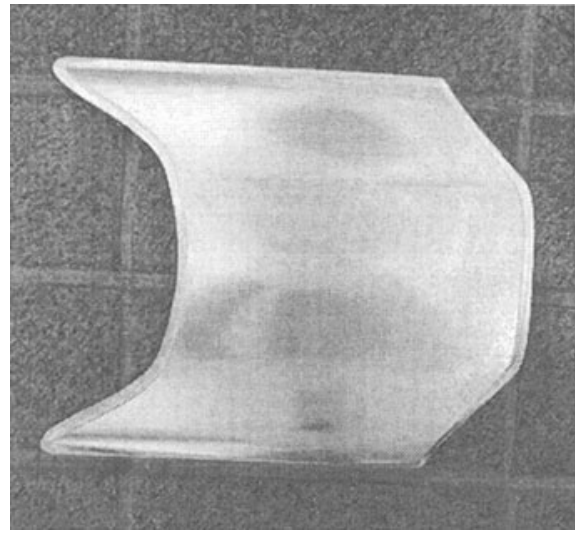

Figure 5: Ball end machined top of the hood.

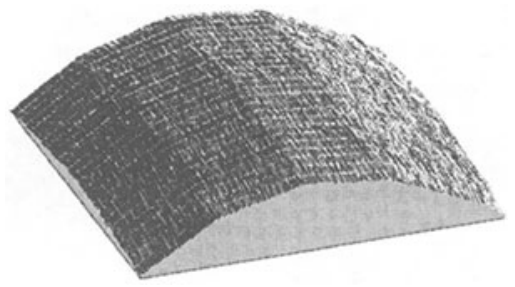

Figure 7: CM machined surface.

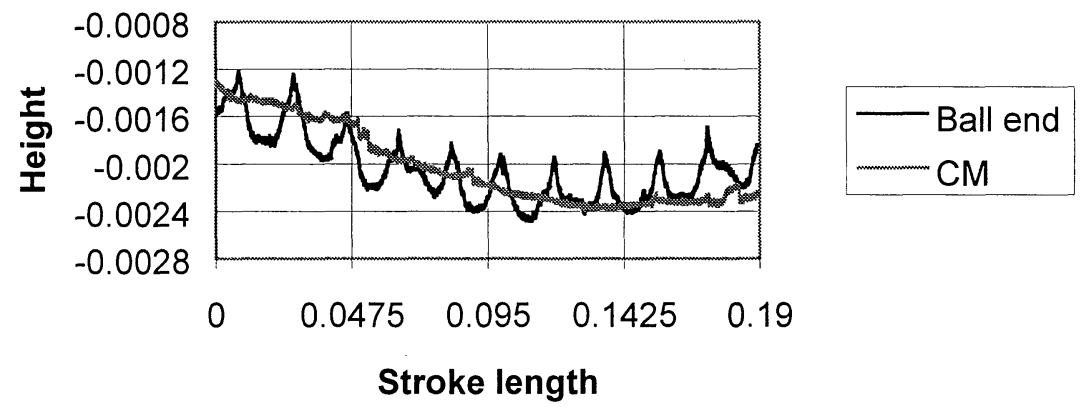

Figure 8: Scallop heights in a concave region.

The surfaces were also compared with respect to tool path density, which directly correlates with machining time. These values are summarized in Table 1. 
Table 1: Tool path density (passes per inch).

\begin{tabular}{|l|l|l|}
\hline & Top & Bottom \\
\hline CM & 10.4 & 9.9 \\
\hline Ball end & 58 & 70 \\
\hline
\end{tabular}

On the top surface, $\mathrm{CM}$ resulted in a nearly 6:1 reduction in tool path density, and on the bottom surface, a greater than 7:1 reduction in tool path density was found.

After machining, all surfaces were inspected for surface roughness $\left(\mathrm{R}_{\mathrm{a}}\right)$. Each surface was inspected at 10 locations, and the results averaged in order to give an average surface roughness for the entire surface. These results are summarized in Table 2.

Table 2: Surface roughness (in).

\begin{tabular}{|l|l|l|}
\hline & Top & Bottom \\
\hline $\mathrm{CM}$ & 36.4 & 36.0 \\
\hline Ball end & 58.4 & 49.4 \\
\hline
\end{tabular}

On each surface, CM resulted in a slightly better overall surface finish. One of the primary goals of $\mathrm{CM}$ is to reduce machining time while also eliminating grinding. This was accomplished on these surfaces. Polishing can generally occur when the surface roughness is below 40 in (Whitehouse, 1994). Above this value, some grinding is still necessary; thus, the ball end machined surfaces would still require light grinding before polishing could be performed.

\section{CONCLUSIONS}

On the surfaces tested here, CM resulted in a smoother surface with a 6 or 7:1 reduction in tool path density. In addition, scallops between adjacent tool paths, while still present with ball end machining, are largely eliminated with $\mathrm{CM}$ due to the precise placement of a filleted or flat end mill on the surface. The surface machined in this study was representative of a stamping die from the automotive industry. Additional research should be performed to investigate the benefits and limitations of CM on other types of geometry, such as composite lay-up tools for the aerospace industry.

\section{REFERENCES}

[1] Mullins, S. H. et. al., "Scallop Elimination Based on Precise 5-axis Tool Placement, Orientation, and Step-Over Calculations", Advances in Design Automation - American Society of Mechanical Engineers, Design Engineering Division (Publication) DE Vol. 65 Pt. 2, pp. 535-544, 1993.

[2] Whitehouse, D. J., Handbook of Surface Metrology, Institute of Physics Publishing, London, England, 1994. 